\title{
Io's Escaping Atmosphere: Continuing the Legacy of Surprise
}

\author{
Nicholas M. Schneider ${ }^{1}$ \\ ${ }^{1}$ LASP, University of Colorado, Boulder CO 80309 USA \\ email: nick.schneider@lasp. colorado.edu
}

\begin{abstract}
The discovery of Io and her fellow Medicean Stars clearly altered the course of science as a whole. It is equally clear that the discovery of Io's tidal heating has altered the course of planetary science. One of the most directly observable consequences of Io's tidal heating is the prodigious escape of a ton per second of volcanically-supplied gases. I will review how studies of Io's escaping atmosphere since 1972 have advanced our deep understanding of Io itself, and helped formulate our perspective on planetary evolution in our solar system and beyond.
\end{abstract}

Keywords. Planets and satellites: Io

\section{Io's unique nature revealed in sodium cloud observations}

Galileo's experience of a new instrument rapidly discovering radical new phenomena has been repeated countless times in the Jovian system, most notably with Io. In 1972, Robert A. Brown was testing a new spectrograph with observations of "known objects", namely the Galilean moons of Jupiter. (These observations took place after Bigg's unexplained discovery of Io-correlated radio emissions from Jupiter (Bigg, 1964) but before the Voyager flyby of Jupiter in 1979.) Brown expected to confirm the performance of the instrument in the form of a simple reflected solar system (Binder and Cruikshank, 1966), but was surprised to find enhanced emission at the location of the sodium D lines near 589nm (Brown, 1972).

The emission was initially thought to be confined to Io's disk, like a glowing atmosphere, but followup observations by Trafton et al. (1974) showed the emission to be extended in a region up to tens of arcseconds from Io. This broad extent explains the discrepancy between Brown's spectra and those of prior observers : Brown's instrument possessed a wide entrance aperture which accepted light from Io and the surrounding region, which enhanced the emission feature relative to Io's reflected continuum. An instrumental design feature implemented to simplify the guiding process had enabled the discovery of the extended, escaping atmosphere of atomic sodium.

Several groups soon confirmed that resonant scattering was the emission process by correlating the brightness of the cloud with the Io's orbital phase. When Io was at its maximum velocity relative to the Sun at maximum elongation, co-moving sodium atoms appeared brighter because they experienced a higher solar flux due to their Doppler shift away from the minimum of the deep solar sodium D-line absorption (Bergstralh et al., 1975; Macy and Trafton, 1975).

Narrow-band imaging then joined high-resolution spectroscopy as an important diagnostic tool (Matson et al., 1978; Goldberg et al. 1980; (Pilcher et al., 1984). These works enabled a new generation of physical models of escape processes whose parameters could be tuned to match observations (e.g., Smyth and McElroy, 1978 and subsequent works). Mendillo et al. (1990) then discovered the cloud extended hundreds of jovian radii away from the planet. These large distances, and the evidence of rapidly-changing features 
near Io (Schneider et al., 1991) led to a new realization that high-speed escape processes, linked to plasma sweeping past Io at $70 \mathrm{~km} / \mathrm{sec}$, were involved.

In parallel, observations and theory were building up an understanding of the plasma torus created by the ionization of these neutral clouds. The plasma torus, in turn, flows onto and around Io's atmosphere, thereby causing escape and completing a positive feedback loop. The structure and behavior of the plasma torus is reviewed in Thomas et al. (2004) and Schneider and Bagenal (2007).

Together these observations have given a solid explanation of the many phenomena exhibited by Io's sodium clouds over a wide range of spatial scales (Figure 1). First, low-speed atoms ejected by sputtering make the slow "banana cloud" which extends insides and slightly forwards from Io (e.g, Smyth et al. 1978). Second, molecular ions are stripped out of the ionosphere, picked up and carried downstream in the torus, where they dissociatively recombine and create the fast sodium "stream" (Wilson and Schneider, 1994). Finally, a jet of sodium atoms is ejected in the anti-Jupiterward direction, created by sodium pickup ions undergoing charge-exchange as they rise out of the atmosphere (Burger et al. 2000). These pheonomena continue to offer a direct means to study Io's escape processes through groundbased observations. Contemporaneous observations of the infrared flux from Io's volcanoes suggests that high volcanic activity enhances energetic escape processes (Mendillo et al. 2004).

\section{The Pervasive Effects of Io's Escaping Atmosphere}

Nearly five decades of observations have stitched together a long list of cause-and-effect relationships which permeate the Jovian system (Figure 2). In the first step, Io's volcanoes release lava, pyroclastic debris and atmospheric gases. The gases form the most spatially and temporally variable atmosphere in the solar system, enhanced in regions of high volcanic outgassing and/or frost sublimation, and minimized in cold polar regions due to condensation. This molecular atmosphere, only weakly held by Io's gravity, begins to escape to space. Subjected to magnetospheric particles and UV radiation, the molecules are broken down into their constituent atoms as the escape continues.

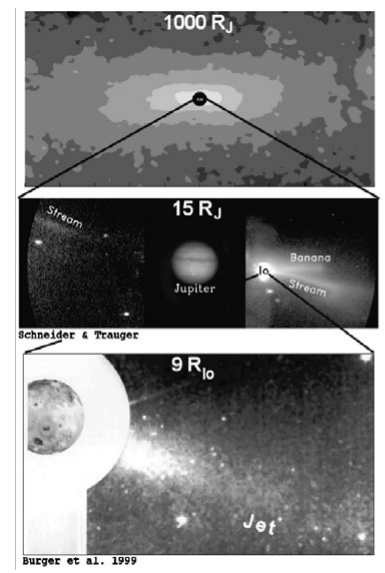

\section{IO SODIUM CLOUDS}

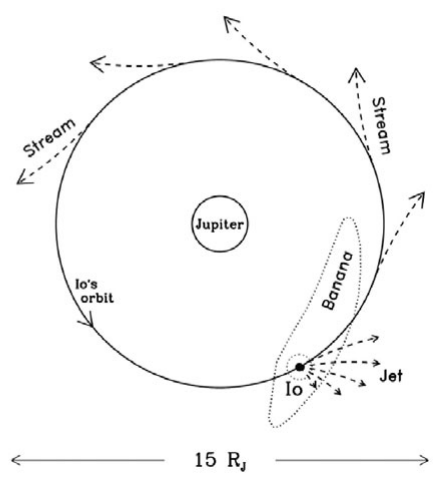

Figure 1. (Left) Io's sodium cloud on three spatial scales, as imaged by groundbased observations of sodium D-line emission. (Right) The features observed at left are explained by the three atmospheric escape processes shown schematically. The Ôbananaõ, jet and cloud are morphological features created by three distinct atmospheric escape processes and are explained in the text. From Schneider and Bagenal (2008) after Thomas et al. (2004) 
Next the escaping gases are ionized and swept into a ring surrounding Jupiter, confined by the jovian magnetic field and co-rotating with the planet. The plasma diffuses inwards and outwards, becoming energized by unknown processes. Some of the plasma becomes capable of traveling along field lines all the way to Jupiter's polar regions, causing aurora. The auroras are sufficiently powerful to dominate sunlight as the prime energy input at high latitudes, driving chemical reactions which create the hydrocarbons which darken the poles at UV wavelengths. Some of the currents driven by precipitating particles give rise to the powerful Jovian radio emissions. Ironically, the driving cause of these phenomena, Io's volcanism, was discovered last, and its most distant effect, the radio emissions, were discovered first. Figure 3 summarizes the profound effects caused by the combination of orbital resonances, tidal heating, atmospheric escape and magnetospheric interactions.

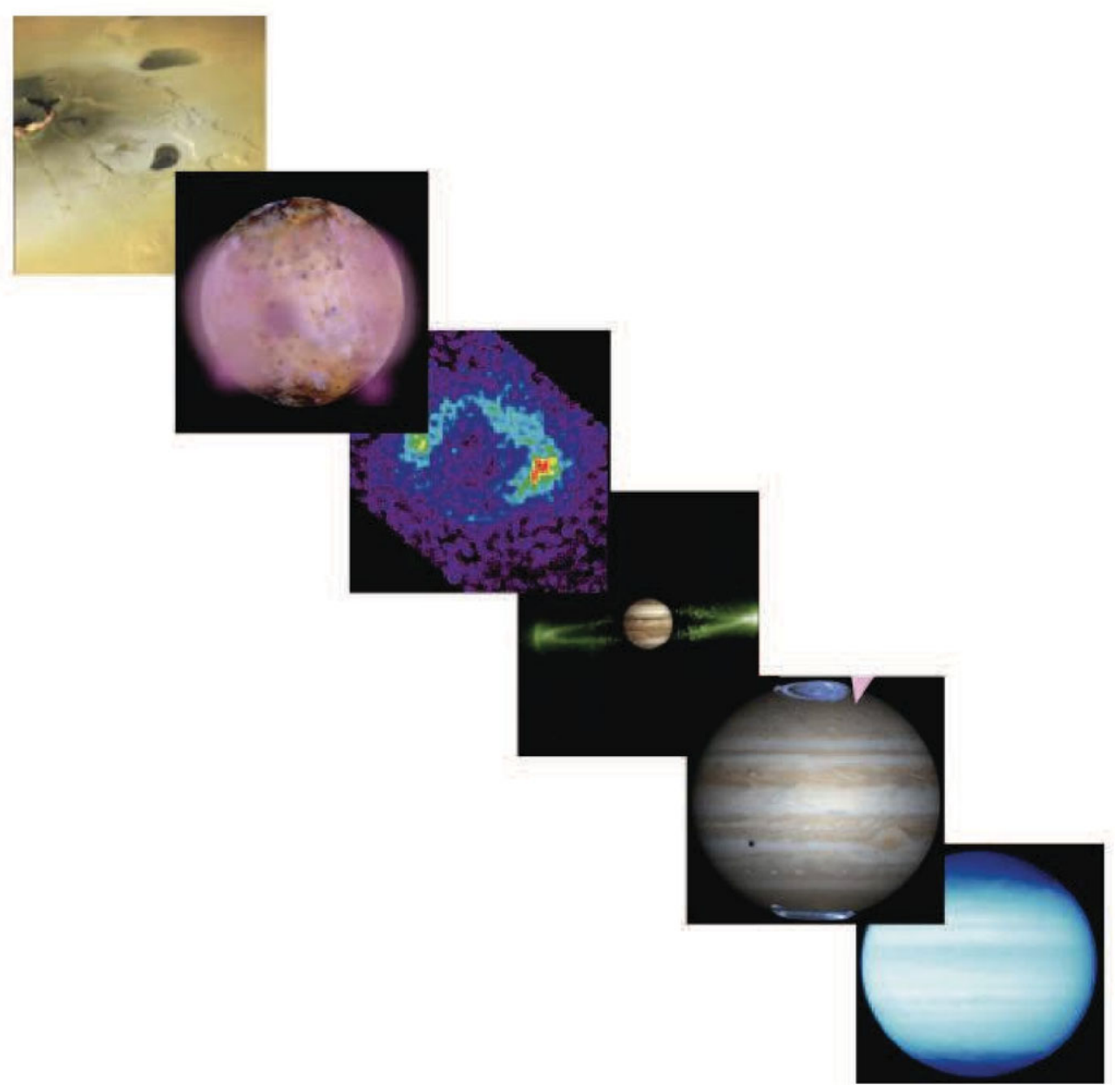

Figure 2. Io's volcanism leads to dramatic changes throughout the Jovian system. The images, respectively, are Io's Tvashtar volcano (imaged by Galileo), an artist's conception of Io's patchy atmosphere overlaid on Io's globe; a Hubble Space Telescope image of Io's corona; a groundbased image of the Io plasma torus, a Hubble Space Telescope image of Jupiter with its UV aurora, and another Hubble Space Telescope image of Jupiter showing polar darkening by auroral energy. 


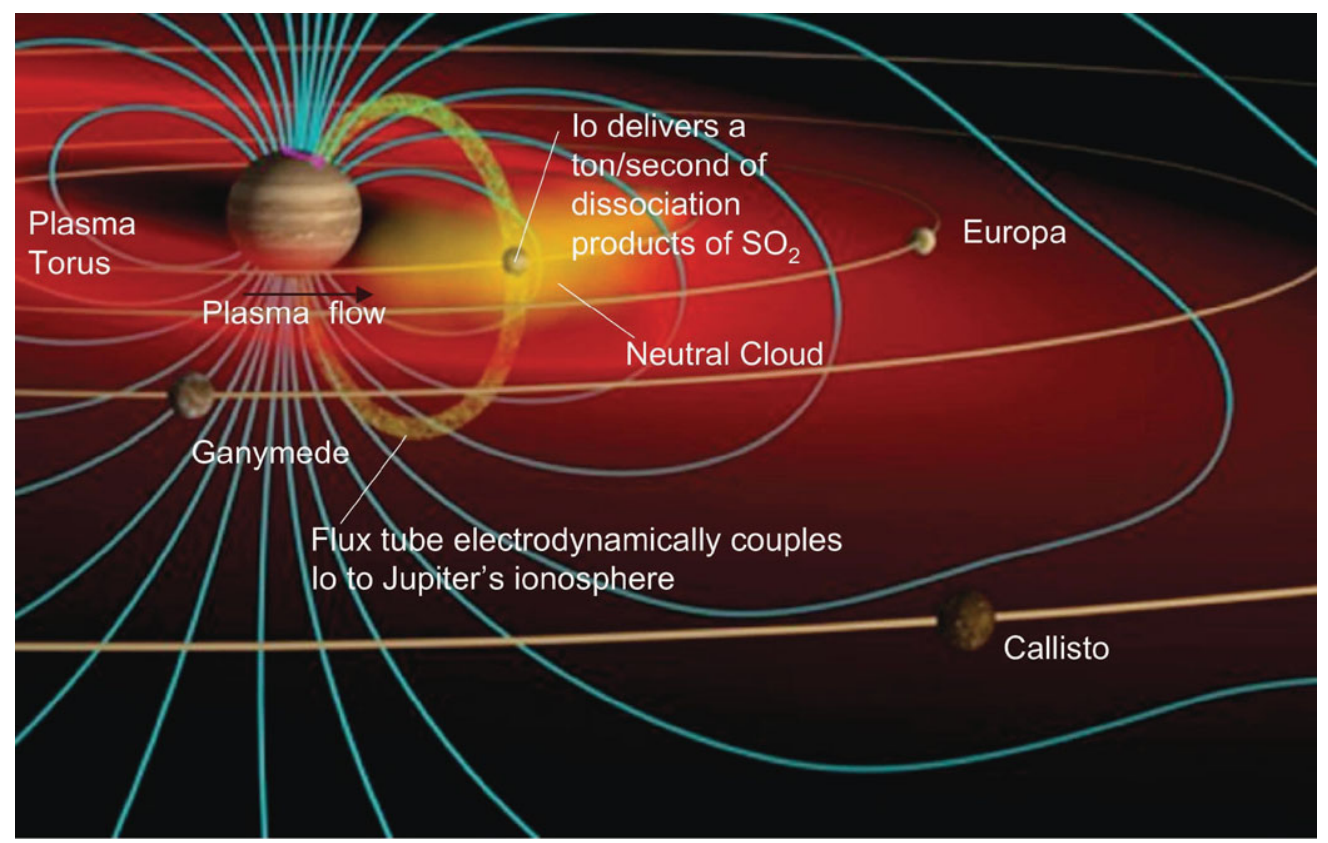

Figure 3. Io's Influences on the Jovian system. Without orbital resonances and tidal heating, these worlds would not affect each other to this dramatic degree.

\section{How Io guides our understanding of atmospheric evolution}

One of the surprises of planetary exploration has been the revelation that planetary atmospheres bear little resemblance to their primordial states. Virtually all objects apart from the jovian planets have undergone significant atmospheric escape. The escape on most worlds acts on timescales of millions or billions of years, and can only be studied by orbital spacecraft and theory. Smaller bodies, by virtue of their lower gravity, undergo more rapid and prominent escape. Io is arguably the archetype of atmospheric escape, losing a ton per second in the current epoch and perhaps $2 \mathrm{~km}$ of its radius over the age of the solar system. Figure 4 shows many of the escape processes that have studied at Io with remote and in situ methods. New escape processes continue to be discovered at Io, and these new processes may prove to be important on other worlds or at at other times. The case of Mars is especially intriguing: NASA's upcoming MAVEN mission ("Mars Atmosphere and Volatile EvolutioN") seeks to measure many of the same nonthermal escape processes observed at Io, with a goal of extrapolating backwards in time to quantify the integrated atmospheric loss of the age of the solar system.

\section{Were the Medicean Stars destined to change planetary science?}

On the occasion of this Galilean anniversary, it's appropriate to speculate on whether the objects within the grasp of Galileo's telescope would inevitably be the ones to profoundly alter our understanding of the planetary processes described above. For example, if Galileo had discovered the moons of Mars, the impact on the Copernican revolution would have been the same. But the lasting impact on planetary science would have been different and arguably diminished. Phobos and Deimos, fascinating objects in their own rights, do not profoundly affect Mars, nor do they serve as the archetype of a transformative process like tidal heating. 


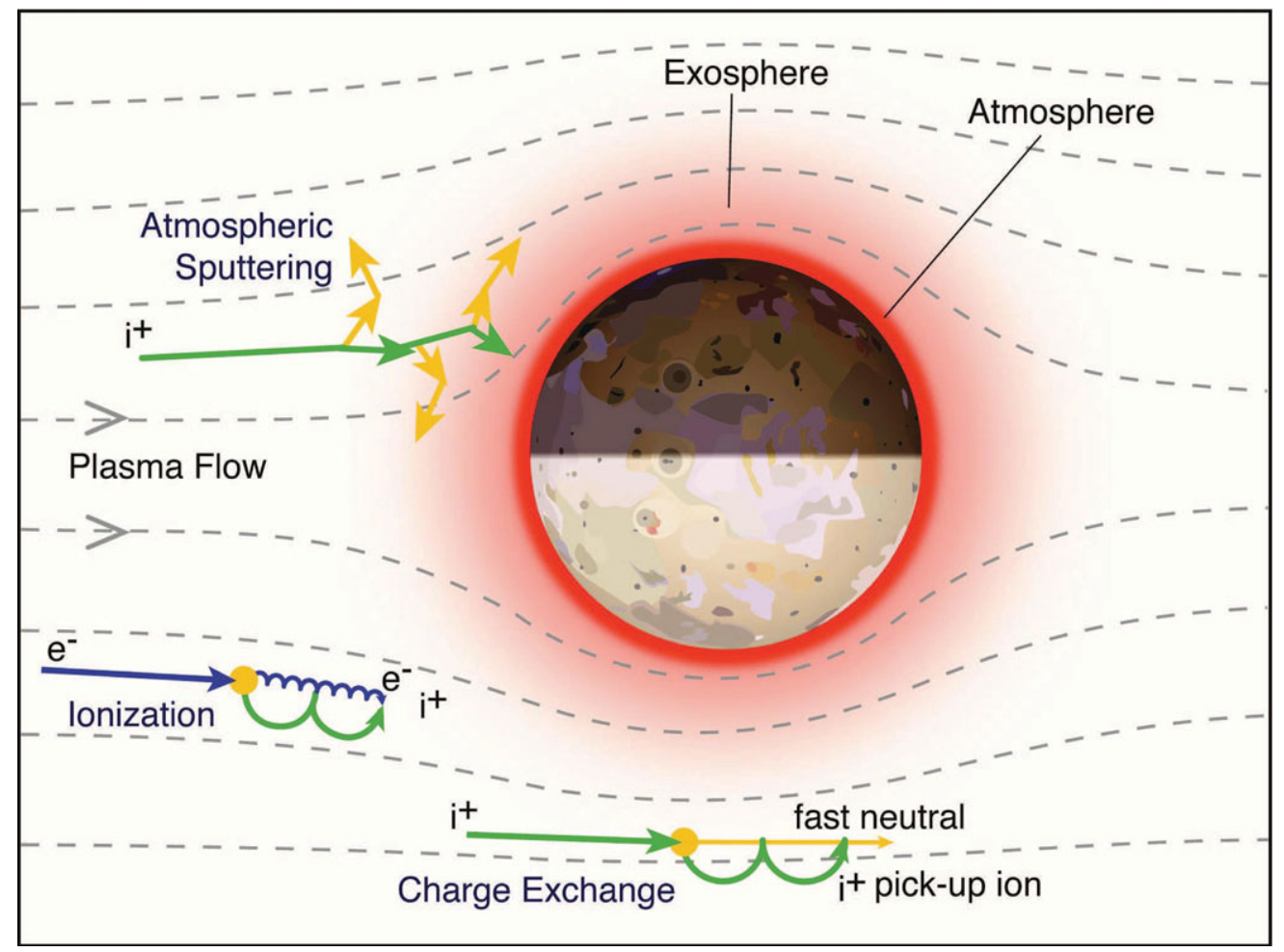

Figure 4. Important plasma/atmosphere interactions near Io. The scale of the gyromotions have been greatly exaggerated: the gyroradius of a pick up oxygen ion is $5 \mathrm{~km}$, much less than Io's radius, and that of an electron is about 40,000 times smaller than the ions. Atmospheric escape occurs at such high rates at Io that many processes are detectable in groundbased observations.

But Galileo's telescope could not have discovered satellites as faint as Phobos and Deimos. His instrument and observing method were most sensitive to bright, fast-moving, numerous moons far enough from their planet to be resolved. The Medicean stars clearly meet these requirements: they are among the largest and most reflective moons in the solar system, and by virtue of their orbit around Jupiter are rendered brighter than satellites of Saturn, Uranus or Neptune. Their location relatively near Jupiter, and Jupiter's great mass, combine to give orbital periods of order days, making their motion easy to detect over the course of a few nights. The fact that there are four moons in the same orbital plane enhanced their detectability, both in their organized appearance and their orbital motions. The Medicean stars could perhaps have been detected even more easily if they orbited closer and faster, but if their orbits had been too small, Galileo's telescope could not have resolved them from Jupiter's glare.

Of all the moons in the outer solar system, only Titan shares any of these criteria for detectability. While its size rivals Callisto's, its greater distance and lower albedo render it roughly ten times fainter than the Medicean stars. And its large orbital distance about a lower-mass planet give a sixteen day period. These facts conspired to prevent Titan's discovery by Huygens until 45 years after Galileo's discovery of the Medicean stars. We can conclude that it was no accident that Jupiter's moons were discovered first. 
One question remains: do the properties that lead to the great detectability of the Medicean stars inevitably lead them to have such fascinating properties? Specifically, is a system of several large, fast-moving satellites relatively close to a very large planet destined to undergo orbital resonances and tidal heating? The answer appears to be "yes". Orbital resonances by definition require two or more satellites, and the Medicean stars offer three. Were Io, Europa and Ganymede destined to become locked in an orbital resonance? Again the answer appears to be affirmative. These objects did not form in resonant orbits, nor is the resonance a coincidence. Each of these objects is large enough and close enough to Jupiter to raise tidal bulges on the planet. Strong tidal torques exerted by Jupiter evolved each of their orbits outwards, with Io moving outwards until achieving resonance with Europa, then the pair evolving outwards until locking into resonance with Ganymede. With eccentricities for all three moons pumped up by their orbital resonance, tidal heating was inevitable. And while their sizes are each large enough to enhance the magnitude of tidal heating to geoloigcally significant levels, the gravity of the moons is too weak to prevent the substantial atmospheric escape which powers the magnetosphere and profoundly alters the Jovian system. While it is conceivable that Jupiter might form with a satellite system containing fewer or smaller moons that might evade resonances and tidal heating, such a system might not have been noticed by Galileo. We are led to conclude that a satellite system within the capabilities of Galileo's telescope in 1610 was destined to undergo the transformative processes of orbital resonances, tidal heating, rapid atmospheric escape and magnetospheric interactions. Surprisingly, the first hints of these effects weren't known for another 350 years, and our understanding of these worlds in many ways remains rudimentary four centuries after their discovery.

This 400th anniversary celebrates the evolution of Galileo's "points of light" into worlds in their own right. By coincidence, recent years have also presented us with new "points of light" to consider: extrasolar planets (Figure 5). These points too will become worlds in their own right in coming years, and some may be controlled by the same processes of orbital resonances, tidal heating, rapid atmospheric escape and magnetospheric interaction. These worlds are sure to continue Galileo's legacy of surprising discoveries.
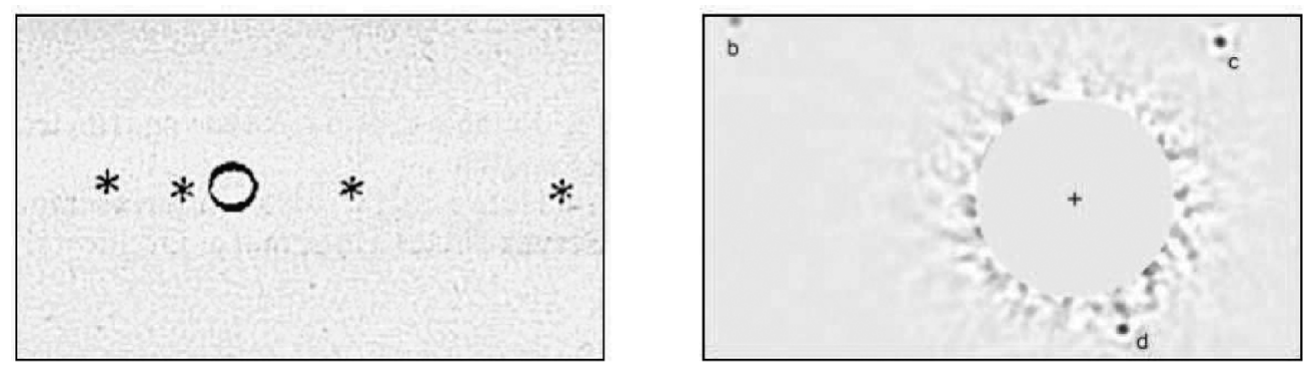

Figure 5. "Points of Light", then and now. The Medicean Stars (left) began as points of light, as shown in a figure from Siderius Nuncius. At right, an infrared image of the region surrounding the star HD8799 reveals three planets, now no more than points of light. Adapted from Marois et al. (2008). 


\section{References}

Bergstralh, J. T., Matson, D. L., \& Johnson, T. V. 1975, Astrophys. J. Lett., 196, L131

Bigg, E. K. 1964, Nature, 203, 1008

Binder, A. B. \& Cruikshank, D. P. 1966, Astrophys. J., 144, 1240

Brown, R. A. 1974, Exploration of the Planetary System, 65, 527

Burger, M. H., Schneider, N. M., \& Wilson, J. K. 2000, Geophys. Res. Lett., 27, 1081

Goldberg, B. A., Mekler, Y., Carlson, R. W., Johnson, T. V., \& Matson, D. L. 1980, Icarus, 44, 305

Goldberg, B. A., Garneau, G. W., \& Lavoie, S. K. 1984, Science, 226, 512

Lellouch, E., Paubert, G., Moses, J. I., Schneider, N. M., \& Strobel, D. F. 2003, Nature, 421, 45

Macy, W. W., Jr. \& Trafton, L. M. 1975, Icarus, 25, 432

Marois, C., Macintosh, B., Barman, T., Zuckerman, B., Song, I., Patience, J., Lafrenière, D., \& Doyon, R. 2008, Science, 322, 1348

Matson, D. L., Goldberg, B. A., Johnson, T. V., \& Carlson, R. W. 1978, Science, 199, 531

Mendillo, M., Baumgardner, J., Flynn, B., \& Hughes, W. J. 1990, Nature, 348, 312

Mendillo, M., Wilson, J., Spencer, J., \& Stansberry, J. 2004, Icarus, 170, 430

Pilcher, C. B., Fertel, J. H., Smyth, W. H., \& Combi, M. R. 1984, Astrophys. J., 287, 427

Schneider, N. M. \& Bagenal, F. 2007, Io After Galileo: A New View of Jupiter's Volcanic Moon, 265

Schneider, N. M., Wilson, J. K., Trauger, J. T., Brown, D. I., Evans, R. W., \& Shemansky, D. E. 1991, Science, 253, 1394

Smyth, W. H. \& McElroy, M. B. 1978, Astrophys. J., 226, 336

Thomas, N., Bagenal, F., Hill, T. W., \& Wilson, J. K. 2004, Jupiter. The Planet, Satellites and Magnetosphere, 561

Trafton, L., Parkinson, T., Macy, W., Jr 1995, Astrophys. J. Lett., 190, L85

Wilson, J. K. \& Schneider, N. M. 1994, Icarus, 111, 31

Wilson, J. K. \& Schneider, N. M. 1999, J. Geophys. Res., 104, 16567 